\title{
Detección de ooquistes de Cryptosporidium parvum (Apicomplexa: Cryptosporidiidae) en ejemplares de cholga Aulacomya ater, extraídas desde la costa de la Región del Bío Bío, Chile
}

\author{
Detection of Cryptosporidium parvum (Apicomplexan: Cryptosporidiidae) oocyst in \\ Aulacomya ater specimen, extracted from to the coast Bio Bio Region, Chile
}

Pilar Suárez R. ${ }^{1}$, María José Yáñez D. ${ }^{2}$, Ítalo Fernández F. y Verónica Madrid V. ${ }^{1}$

\begin{abstract}
'Laboratorio de Parasitología. Departamento de Microbiología, Facultad de Ciencias Biológicas, Universidad de Concepción. Concepción, Chile.
${ }^{2}$ Laboratorio de Entomología Aplicada, Departamento de Zoología, Facultad de Ciencias Naturales y Oceanográficas, Universidad de Concepción. Concepción, Chile.

Los autores declaran ausencia de conflictos de interés

Financiamiento: Facultad de Ciencias Biológicas. Universidad de Concepción.
\end{abstract}

Recibido: 2 de octubre de 2019 / Aceptado: 1 de abril de 2020

\section{Resumen}

Introducción: El bivalvo Aulacomya ater (cholga), es uno de los moluscos de mayor consumo en la población chilena. Sin embargo, existe evidencia de contaminación fecal hídrica provocada por los cauces que llegan al mar, aumentando la probabilidad de contaminación por Cryptosporidium parvum, el que genera criptosporidiosis en el ser humano. Objetivo: Determinar la presencia de C. parvum en cholgas extraídas desde la Región del Bío Bío (Chile). Material y Métodos: Se seleccionaron 55 cholgas provenientes de un centro de cultivo y de un banco natural de extracción. Estas muestras, fueron procesadas en el laboratorio y se evaluó la presencia de elementos ácido-alcohol resistentes. Las muestras positivas, se analizaron por inmunofluorescencia directa, con anticuerpo específicos contra $C$. parvum. Resultados: $16,4 \%$ del total de las muestras tenían ooquistes de C. parvum. Conclusiones: Por primera vez se describe $C$. parvum en $A$. ater provenientes de las costas chilenas, siendo este molusco un posible vehículo de transmisión de criptosporidiosis a la población y a sus animales depredadores. Además, la presencia de C. parvum refleja la contaminación fecal hídrica en las costas evaluadas. Actualmente estamos monitoreando otras zonas de extracción de este molusco.

Palabras clave: Cryptosporidium parvum; Aulacomya ater; cholga; enfermedad de transmisión alimentaria (ETA); Chile; parásitos.

\begin{abstract}
Background: The bivalve Aulacomya ater (cholga), is one of the most consumed mollusks by the population. However, there is evidence of fecal water contamination caused by causes that affect the sea, increasing the probability of contamination by the Cryptosporidium parvum, which generates cryptosporidiosis in people. Aim: To determine the presence of C. parvum in cholga extracted from the Bio Bio Region (Chile). Methods: Fifty-five cholgas were selected from a cultivation center and a natural extraction bank. These samples were processed in the laboratory and the presence of acid-alcohol resistant elements was evaluated. Positive samples were analyzed by direct immunofluorescence with anti-C. parvum antibody. Results: $16.4 \%$ of the total samples were affected by the oocysts of C.parvum. Conclusions: For the first time we described C. parvum in A. ater from the Chilean coast, being this mollusk a possible vehicle for transmission of cryptosporidiosis to the population and their predatory animals. Furthermore, the presence of C. parvum reflects fecal water contamination on the evaluated coasts. We are currently monitoring other extraction areas for this mollusk.

Keywords: Cryptosporidium parvum; Aulacomya ater; cholga; foodborne disease; Chile; parasite.
\end{abstract}

\footnotetext{
Correspondencia a:

Pilar Suárez Roa

pilarsuarez@udec.cl
} 


\section{Introducción}

L os moluscos bivalvos son un grupo de invertebrados, principalmente marinos, que constituyen recursos de importancia comercial en Chile. Estas especies se alimentan mediante nutrientes que obtienen al filtrar el agua a través de sus branquias (entre 20 y 100 litros de agua al día). Durante este proceso, los moluscos pueden retener microorganismos patógenos tales como bacterias, virus y protozoos presentes en el ambiente, los que se adhieren en el mucus secretado en esta estructura, o bien, pueden pasar hacia su tracto digestivo, acumulándose a lo largo del tiempo ${ }^{1}$. Algunos autores han reportado la presencia de distintos protozoos patógenos en bivalvos de aguas dulce y/o salada ${ }^{2-5}$, dentro de los cuales se ha identificado la presencia del coccidio intestinal Cryptosporidium sp., patógeno oportunista del ser humano.

A la fecha, se han identificado más de 20 especies del género Cryptosporidium, cuyos reservorios son el ser humano y/o diversos taxa animales ${ }^{6,7}$. En Chile, la especie prevalente en el ser humano es Cryptosporidium parvum, la que ha sido observada con mayor frecuencia en exámenes de deposición provenientes de pacientes inmunocomprometidos que presentaban cuadros de diarrea crónica ${ }^{8}$. Cryptosporidium parvum tiene como principal reservorio a bovinos, y se transmite a diferentes especies animales mediante fecalismo directo ${ }^{9,10}$. En seres humanos, puede ocasionar cuadros de diarrea aguda en pacientes inmunocompetentes y de diarrea crónica en hospederos inmunocomprometidos, para los cuales no existe un tratamiento efectivo ${ }^{11,12}$. El ooquiste, su forma infectante, es resistente a las condiciones ambientales, a las concentraciones normales de cloración del agua y desinfectantes comunes, entre otros. Bastan 10 ooquistes para generar una infección ${ }^{7}$, dosis infectante mínima que lo convierte en un microorganismo potencialmente transmisible hacia la población que consume bivalvos crudos o insuficientemente cocidos, contaminados con deposiciones humanas y/o animales ${ }^{13,14}$.

En la Región del Bío Bío, numerosos ríos y canales se encuentran contaminados con heces de origen humano $\mathrm{y}$ animal debido a que, durante su trayecto reciben descargas de vertederos de aguas servidas, alcantarillados y bostas de producciones animales, vehiculizándose hasta el mar y, por lo tanto, contaminando bancos naturales y cultivos artesanales de bivalvos. Además, corrientes marinas pueden arrastrar este material hacia zonas de producción y extracción de bivalvos. Estudios recientes, atribuyen a estos moluscos la utilidad de ser una herramienta idónea de monitoreo para la evaluación de calidad de agua ${ }^{15}$.

Al día de hoy, no se cuenta con antecedentes sobre la presencia de $C$. parvum en moluscos bivalvos de consumo habitual en Chile, por lo que, el objetivo de esta investigación fue determinar la presencia de C. parvum en muestras de bivalvos obtenidos desde bancos naturales y de centros de cultivo artesanales, ubicados en la zona costera, de la Región del Bío Bío (Chile).

\section{Material y Método}

\section{Obtención de las muestras}

La pesquisa de Cryptosporidium sp. se efectuó en ejemplares de la cholga Aulacomya ater Molina 1782, una de las especies más consumidas por la población. Veinticinco moluscos fueron extraídos desde un centro de cultivo y 30, desde un banco natural. Ambos sectores se encuentran distanciados por 9,5 Km. En ambas localizaciones desembocan cursos de agua dulce (micro esteros, canales, ríos pequeños, etc.) en cuyo trayecto recorren una zona principalmente vinculada a la agricultura y ganadería doméstica. Además, existen numerosas fuentes de aguas servidas que son vertidas directamente a estos cauces llevando este material hacia el mar.

Los especímenes fueron seleccionados de acuerdo a su tamaño (10 a $12 \mathrm{~cm}$ ) según el criterio señalado por Food and Drug Administration de E.U.A. ${ }^{16}$. Éstos se transportaron dentro de un recipiente hermético a $4{ }^{\circ} \mathrm{C}$, al Laboratorio de Parasitología, Facultad de Ciencias Biológicas, Universidad de Concepción, para su posterior procesamiento. En este lugar los especímenes fueron identificados taxonómicamente, de acuerdo a lo señalado en bibliografía ${ }^{17}$.

\section{Procesamiento de los ejemplares}

Con posterioridad a la apertura de las valvas se hizo disección para extraer hepatopáncreas y branquias. Ambos órganos fueron macerados con mortero agregando $15 \mathrm{ml}$ de tampón fosfato salino (PBS, $\mathrm{pH}$ 7.4) para su homogenización. Este material se dejó decantar por 12 horas a temperatura de $20{ }^{\circ} \mathrm{C}$. Al día siguiente, y con el objetivo de pesquisar ooquistes de Cryptosporidium sp., se eliminó el sobrenadante y desde el sedimento se extrajo una alícuota para confeccionar un frotis que fue teñido con tinción Ziehl-Neelsen (TZN) según el protocolo ${ }^{18}$. Todos los sedimentos se conservaron a $4^{\circ} \mathrm{C}$ en etanol al $70 \% \mathrm{v} / \mathrm{v}$ para su ulterior estudio.

\section{Microscopía de fluorescencia}

Las muestras positivas con TZN se estudiaron mediante inmunofluorescencia directa (IFD) con el fin de identificar C. parvum. Para ello, se utilizó el anticuerpo comercial DynabeadsTM anti Cryptosporidium (J27E) ${ }^{\circledR}$ 
FITC de acuerdo a las especificaciones técnicas del fabricante (Thermo Fisher, EE.UU). Este anticuerpo no presenta reacción cruzada con otros protozoos parasitos ni otras especies del genero Cryptosporidium (Thermo Fisher, EE.UU).

Las muestras fueron observadas en microscopio de Epifluorescencia (Zeiss Axioplan 2) con objetivo de 40x, el que se encontraba conectado a una cámara digital (Nikon Digital Camera DXM 12000). Para su visualización en multimedia se utilizó un software asociado (Nikon ACT-1 Versión 2).

\section{Resultados}

Se analizaron 55 muestras de $A$. ater, provenientes de un centros de cultivos o de un banco natural (Tabla 1). Se identifico ooquistes del género Cryptosporidium sp. por TZN. El 16,4\% de las muestras fue positivo, correspondiendo a $12,7 \%$ las muestras que venían desde el banco natural y $3,6 \%$ a las de zona de centro de cultivo artesanal (Figuras 1 a 3). Posteriormente, estas muestras fueron analizadas por IFD para identificar especie de C. parvum. En 14,5\% se detectó estos ooquistes en las muestras (Figuras 4 a 6), lo que corresponde a 10,9\% de muestras del banco natural y $3,6 \%$ de centro de cultivo. Estas muestras también fueron evaluadas por reacción de polimerasa en cadena (RPC) en tiempo real; sin embargo, no fue posible la extracción de ADN. Cabe destacar que la especificidad del anticuerpo permite identificar la especie C. parvum con alta selectividad.

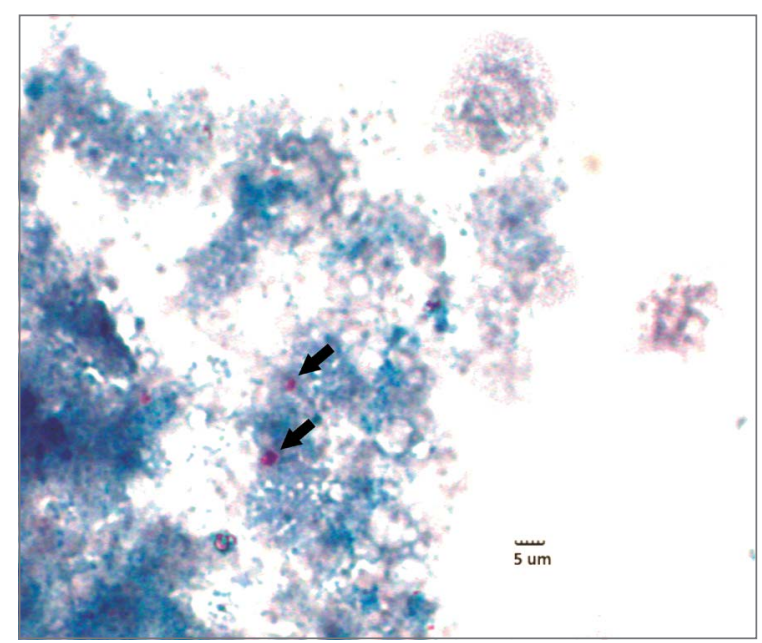

Figura 2. Ooquiste de Cryptosporidium sp. (flecha negra) en muestras de $A$. ater, provenientes de banco natural, aumento 40x (en TZN).

\begin{tabular}{|c|c|c|c|c|c|c|}
\hline Método & $\begin{array}{c}\text { Total muestras } \\
\text { (55) }\end{array}$ & $(\%)$ & $\begin{array}{c}\text { Banco natural } \\
(30)\end{array}$ & $(\%)$ & $\begin{array}{l}\text { Centro de } \\
\text { cultivo (25) }\end{array}$ & $(\%)$ \\
\hline TZN (+) & 9 & 16,4 & 7 & 12,7 & 2 & 3,6 \\
\hline $\operatorname{IFD}(+)$ & 8 & 14,5 & 6 & 10,9 & 2 & 3,6 \\
\hline \multicolumn{7}{|c|}{$\begin{array}{l}\text { TZN (+): muestras Tinción Ziehl Neelsen positivos (Cryptosporidium sp.), IFD (+): muestras cuya } \\
\text { inmunofluorescencia directa es positivas (Cryptosporidium parvum), (\%): porcentaje de muestras } \\
\text { positivas. }\end{array}$} \\
\hline
\end{tabular}

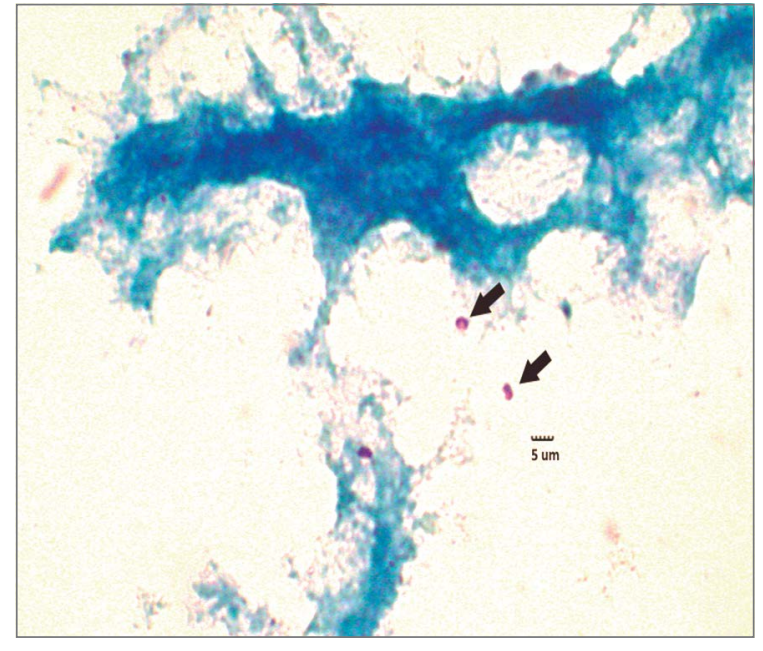

Figura 1. Ooquiste de Cryptosporidium sp. (flecha negra) en muestras de deposición obtenida desde paciente con criptosporidiosis, aumento 40x (Control positivo de TZN).

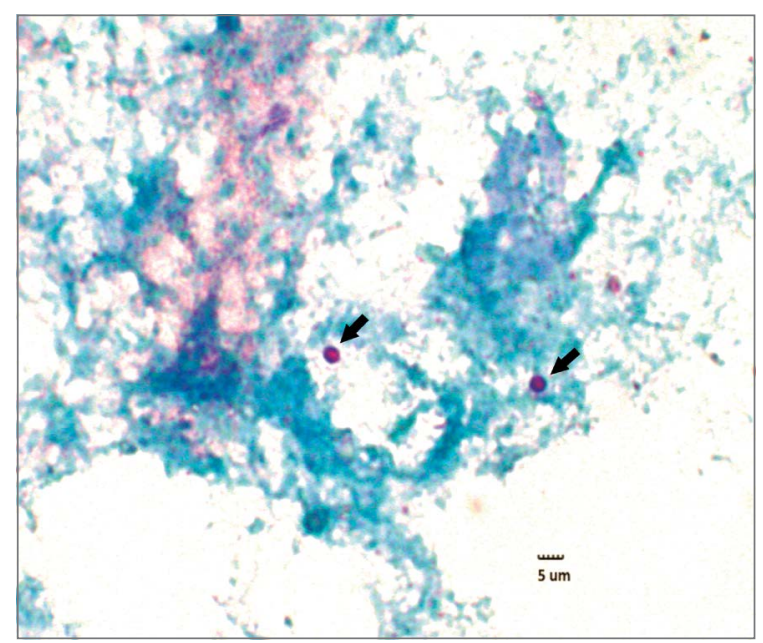

Figura 3. Ooquiste de Cryptosporidium sp. (flecha negra) en muestras de $A$. ater, provenientes de centro de cultivo, aumento 40x (en TNZ). 
Figura 4. Ooquiste de C. parvum, aumento 40x (Control positivo IFD, Thermo Fisher).
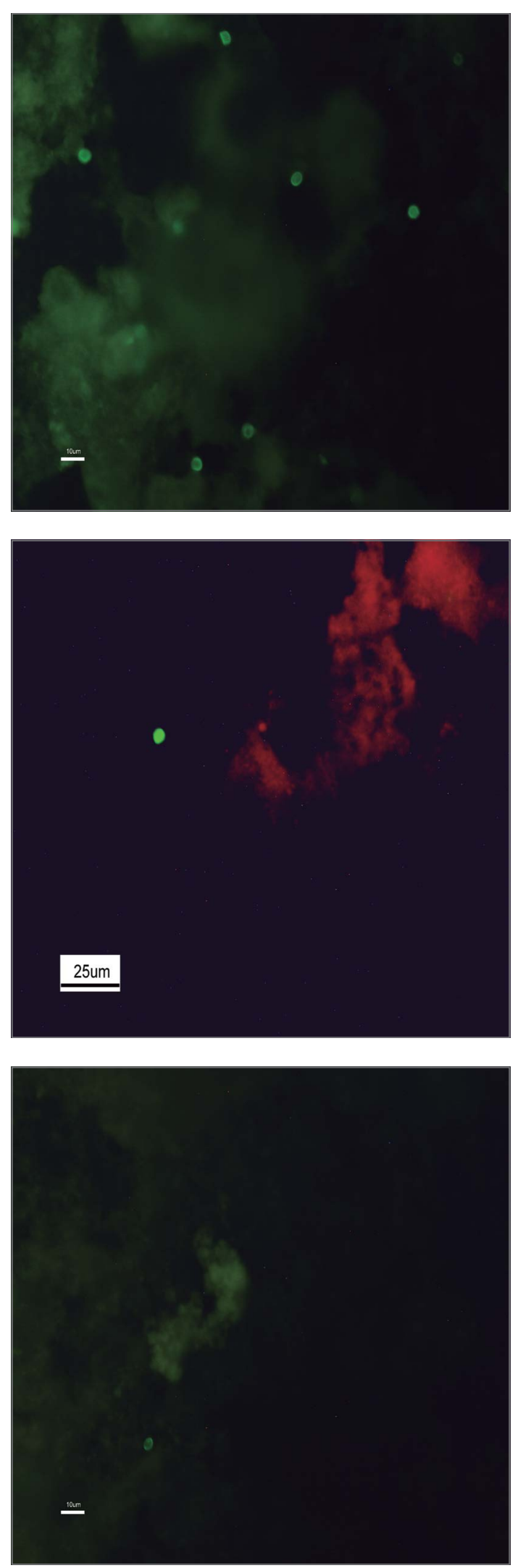

\section{Discusión}

Cryptosporidium sp. es reconocido mundialmente como un patógeno que afecta la calidad del agua en países desarrollados y en vías de desarrollo ${ }^{19}$. Su diseminación y sobrevivencia se ve favorecida por las características físico-morfológicas del ooquiste, como su tamaño (4 a $6 \mu \mathrm{m})$ y composición de su pared, la que le confiere tolerancia a ambientes adversos.

Este protozoo es difícil de controlar debido a su baja dosis infectante, a su amplia gama de especies hospedadoras y a un prolongado período de patencia. Por ello, el manejo de este parasitismo implica identificar todas las probables fuentes de infección para aplicar las medidas profilácticas respectivas, sobre todo para personas inmunodeprimidas. Además, de que los costos asociados al tratamiento son elevados y no son efectivos ${ }^{11,12,20}$.

Se pesquisó ooquistes de C.parvum en las muestras provenientes del centro de cultivo y del banco natural, con un porcentaje de 3,6 y $10,9 \%$, respectivamente. Esta diferencia puede tener su origen en las diferencias geográficas de ambos terrenos. El centro de cultivo se encuentra en una caleta donde desemboca un estero, el que se origina desde un río que pasa por zonas de criaderos de distintos animales (vacas y cerdos, principalmente). El estero, antes de desembocar en la caleta, recorre un tramo de $7 \mathrm{~km}$, sirviendo como fuente de extracción de agua para el consumo humano. Por otro lado, corriente abajo del estero los lugareños vierten las aguas lluvias de la misma localidad y se desconoce la posibilidad de vertimientos de aguas servidas ${ }^{21}$.

A su vez, el banco natural, se ubica en una bahía ampliada alejada de la costa. En la playa, frente al banco natural, existe descargas de aguas contaminadas, que puede ser la principal fuente de C. parvum. Este fenómeno ya ha sido observado en Canadá, España y México ${ }^{22,23,24}$ donde se encontró contaminación por Cryptosporidium sp. en áreas de cosecha de moluscos bivalvos de interés comercial. Además, para ambas zonas la cercanía de núcleos urbanos implica la realización de numerosas actividades recreacionales, entrada de escorrentía de origen fluvial. Por otro lado, existen deficiencias en tratamientos de aguas residuales que se vierten al mar y la presencia de numerosos perros en la playa ${ }^{25}$, hospederos definitivos de estos parásitos, los que podrían incidir en la contaminación de estos sectores costeros ${ }^{26}$.

La cholga como alimento, es el segundo producto bivalvo más consumido por la población después del chorito ${ }^{27}$. Si bien se describen formas de descontaminación de bivalvos a nivel industrial ${ }^{14}$, la captura artesanal no mantiene estas prácticas, porque ofrece productos frescos. Por lo cual, se necesita reforzar las medidas profilácticas individuales para evitar la infección, ya sea con campañas dirigidas a las cocinerías de la zona y a
Figura 6. Ooquiste de C. parvum en muestras de $A$. ater, provenientes de centro de cultivo, aumento 40x (en IFD). 
áreas con polución y acumular en sus tejidos sustancias tóxicas y microorganismos de forma proporcional a las concentraciones ambientales, como los moluscos Mytilus galloprovincialis y Corbicula japonica ${ }^{31,34-38}$, representando un método alternativo a las técnicas tradicionales para detectar enteropatógenos en muestras de agua ${ }^{38}$.

Algunas limitaciones del uso de bioindicadores son los muestreos que consumen tiempo, la información es cualitativa y se requiere experiencia para la identificación taxonómica ${ }^{39}$; además, en algunos países es establecen períodos de veda debido a que algunas especies están protegidas $^{38}$

En conclusión, se describe por primera vez la identificación de C. parvum en A. ater de las costas chilenas. Se postula que $A$. ater se comportaría como un vehículo de transmisión de criptosporidiosis. Por lo tanto, es necesario revisar la cobertura del alcantarillado en las zonas de cultivo de estos bivalvos, y hacer monitoreo de las corrientes marinas. Además, se deben incluir nuevas políticas que ayuden al tratamiento de aguas servidas ya que los procedimientos para la descontaminación de elementos parasitarios son diferentes a los usados para bacterias y virus. Se establece a $A$. ater como un organismo de monitoreo de contaminación ambiental para $C$. parvum. Con base a los resultados presentados, el estudio de la contaminación fecal humana y/o animal usando la detección de C. parvum en A. ater, en zonas costeras, será el foco de nuestros futuros estudios.

Agradecimientos. A la Facultad de Ciencias Biológicas de la Universidad de Concepción por el financiamiento entregado para la realización de este estudio. Al Profesor Dr. Hernán Montecinos P., por facilitarnos el equipo de microscopia de epifluorescencia.

\section{Referencias bibliográficas}

1.- DiGirolamo R, Liston J, Matches J. Ionic bonding, the mechanism of viral uptake by shellfish mucus. Appl Environ Microbiol 1977; 33 (1): 19-25. PMID: 13712.

2.- Marangi M, Giangaspero A, Lacasella V, Lonigro A, Gasser R B. Multiplex PCR for the detection and quantification of zoonotic taxa of Giardia, Cryptosporidium and Toxoplasma in wastewater and mussels. Mol Cell Probes 2015; 29: 122-5. doi: 10.1016/j.mcp.2015.01.001.

3.- Słodkowicz-Kowalska A, Majewska A C, Rzymski P, Skrzypczak L, Werner A. Human waterborne protozoan parasites in freshwater bivalves (Anodonta anatina and Unio tumidus) as potential indicators of fecal pollution in urban reservoir. Limnologica 2015; 51: 32-6. doi: 10.1007/s11356-0178652-y.
4.- Ghozzi K, Marangi M, Papini R, Lahmar I, Challouf R, Houas N, et al. First report of Tunisian coastal water contamination by protozoan parasites using mollusk bivalves as biological indicators. Mar Pollut Bull 2017; 117: 197-202. doi: 10.1016/j. marpolbul.2017.01.057.

5.- Coupe A, Howe1 L, Burrows E, Sine A, Pita A, Velathanthiri N, et al. First report of Toxoplasma gondii sporulated oocysts and Giardia duodenalis in commercial green-lipped mussels (Perna canaliculus) in New Zealand. Parasitol Res 2018; 117: 1453-63. doi: 10.1007/ s00436-018-5832-8.

6.- Smith H, Nichols R. Cryptosporidium: Detection in water and food. Exp Parasitol 2010; 124: 61-79. doi: 10.1016/j. exppara.2009.05.014.

7.- Ryan U, Hijjawi N, Xiao L. Foodborne criptosporidiosis. Int J Parasitol 2018; 48: 1-12. doi: 10.1016/j.jpara.2017.09.004.

8.- Neira P, Muñoz N, Wilson G, Barthel E, Rosales M J, Henríquez C. Especies de Cryptosporidium en pacientes inmunodeficientes e inmunocompetentes de Valparaíso. Estudio descriptivo. Rev Chil Infect 2012; 29 (1): 63-71. doi: 10.4067/S071610182012000100011.

9.- Weitz J C, Tassara R. Cryptosporidiosis. Atías A. Parasitología Médica. $3^{\circ} \mathrm{Ed}$, Editorial Mediterráneo. Santiago, Chile. 1998; pp 146-51.

10.- Acha P, Szyfres B. Zoonosis y enfermedades transmisibles comunes al hombre y a los animales. 3 Edición, Washintong, Estados Unidos. 2003; 3: pp 23-6.

11.- Abubakar I, Aliyu S H, Arumugam C, Usman N K, Hunter P R. Treatment of cryptosporidiosis in immunocompromised individuals: systematic review and meta- 
analysis. Br J Clin Pharmacol 63;4: 387-93. doi: 10.1111/j.1365-2125.2007.02873.x

12.- Sparks H, Nair G, Castellanos-González A, White C A. Treatment of Cryptosporidium: What we know, gaps, and the way forward. Curr Trop Med Rep 2015; 2 (3): 181-7. doi:10.1007/s40475-015-0056-9.

13.- Gómez Couso H, Freire Santos F, Hernández G, Arés M. A histological study of the transit of Cryptosporidium parvum oocysts through clams (Tapes decussatus). Int J Food Microbiol. 2005; 102: 57-62. doi:10.1016/j. ijfoodmicro.2004.12.002.

14.- Sutthikornchai C, Popruk S, Chumpolbanchorn K, Sukhumavas W, Sukthana Y. Oyster is an effective transmission vehicle for Cryptosporidium infection in human. Asian Pac J Trop Med. 2016; 9(6): 562-6. doi: 10.1016/j. apjtm.2016.04.018.

15.- Graczyk T K, Bruce D, Lucy F, Minchin D, Tamang L, Moura L, et al. Human waterborne parasites in zebra mussels (Dreissena polymorpha) from the Shannon River drainage area, Ireland. Parasitol Res 2004; 93: 385-91. doi:10.1007/s00436-004-1142-4.

16.- Food and Drug Administration (FDA). Compliance Program Guidance Manual. Chapter 03-Foodborne biological hazards. Import Seafood Products Compliance Program. 2010; 63 pp. https://www.fda.gov/media/78911/ download

17.- Aldea C, Valdovinos C. Moluscos del intermareal rocoso del centro-sur de Chile $\left(36^{\circ}\right.$ - $\left.38^{\circ} \mathrm{s}\right)$ : Taxonomía y clave de identificación. Gayana 2005; 69 (2): 364-96. doi: 10.4067/ S0717-65382005000200014

18.- Prescott L M, Harley J P, Klein D A. Microbiología. $5^{\circ}$ Edición, Editorial McGrawHill. Madrid, España. 2004; 1236 pp.

19.- Medema G, Teunis P, Blokker M, Deere D, Davison A, Charles P, Loret JF. Guidelines for drinking water quality: Cryptosporidium. Organización Mundial de la Salud (OMS). 2006.

20.- Corso P S, Kramer M H, Blair K A, Addiss D G, Davis J P, Haddix A C. Cost of Illness in the 1993 waterborne Cryptosporidium outbreak, Milwaukee, Wisconsin. Emerg Infect Dis 2003; 9 (4): 426-31. doi:10.3201/eid0904.020417.

21.- Sobarzo M. Caracterización de la circulación de Bahía Concepción, Chile: un análisis temporal en el dominio de la frecuencia. Tesis de Magister en Ciencias, Mención
Oceanografía, Facultad de Ciencias Naturales y Oceanográficas, Departamento de Oceanografía, Universidad de Concepción. Concepción, Chile. 1993.

22.- Gómez Couso H, Méndez F, Castro J A, Ares E. Cryptosporidium contamination in harvesting areas of bivalve molluscs. J Food Prot 2006; 69 (1): 185-90. doi: 10.4315/0362028x-69.1.185.

23.- Cáceres J, Vásquez R. Manual de buenas prácticas de manejo para el cultivo de moluscos bivalvos. OIRSA-OSPESCA 2014; pp 146.

24.- Aguirre J, Greenwood S J, McClure J T, Davidson J, Sánchez J. Effects of rain events on Cryptosporidium spp. levels in commercial shellfish zones in the Hillsborough River, Prince Edward Island, Canada. Food Waterborne Parasitol 2016; 5: 7-13. doi: 10.1016/j.fawpar.2016.08.003

25.- Luzio A, Belmar P, Troncoso I, Luzio P, Jara A, Fernández I. Formas parasitarias de importancia zoonótica, encontradas en heces de perros recolectadas desde plazas y parques públicos de la ciudad de Los Ángeles, Región del Bío Bío, Chile. Rev Chilena Infectol 2015; 32 (4): 403 7. doi:10.4067/S0716-10182015000500006.

26.- González O. Cryptosporidium y Giardia en moluscos bivalvos de interés comercial. Memoria del Trabajo Fin de Máster. Departamento de Microbiología y Parasitología, Facultad de Farmacia, Universidad de Santiago de Compostela. 2014; pp 100.

27.- Servicio Nacional de Pesca y Acuicultura (SERNAPESCA). Anuario Estadístico de pesca 2011. Ministerio de Economía, Fomento y Turismo, Chile. 2011.

28.- Rivera N, Parra G, Gutierrez G, Cisternas M, Mella S. Estudio preliminar sobre enteroparasitosis y Cryptosporidium sp. en niños de consultorios urbanos y un centro de rehabilitación nutricional de Concepción, Chile 1994. Parasitol al Día 2015; 19: 137-9.

29.- Izumi T, Itoh Y, Yagita K, Endo T, Ohyama T. Brackish water benthic shellfish (Corbicula japonica) as a biological indicator for Cryptosporidium parvum oocysts in river water. Bull Environ Contam Toxicol 2004; 72: 29-37. doi: 10.1007/s00128-003-0237-4.

30.- Pedrero S P. Detección y caracterización de virus patógenos humanos en muestras ambientales y moluscos bivalvos. Tesis para optar al grado de Doctor. Facultad de Biología,
Departamento de Microbiología, Universidad de Barcelona. Barcelona, España.2001; pp 280.

31.- González O. Cryptosporidium y Giardia en moluscos bivalvos de interés comercial. Memoria del Trabajo Fin de Máster. Departamento de Microbiología y Parasitología Facultad de Farmacia, Universidad de Santiago de Compostela. 2014. pp 100.

32.- Giangaspero A, Cirillo R, Lacasella V, Lonigro A, Marangi M, Cavallo P, et al. Giardia and Cryptosporidium inflowing water and harvested shellfish in a lagoon in southern Italy. Parasitol Int 2005; 58: 12-7. doi:10.1016/j. parint.2008.07.003.

33.- Graczyk T, Fayer R, Lewis E, Trout J, Farley C. Cryptosporidium oocysts in Bent mussels (Ischadium recurvum) in the Chesapeake Bay. Parasitol Res 1999; 85: 518-21. doi: 10.1007/ s004360050590.

34.- Graczyk T, Conn D, Marcogliese D, Grazczyk $\mathrm{H}$, Lafontaine Y. Accumulation of human waterborne parasites by zebra (Dreissena polymorpha) and Asian freshwater clams (Corbicula fluminea). Parasitol Res 2003; 89: 107-12. doi: 10.1007/s00436-002-0729-x.

35.- Izumi T, Yagita K, Endo T, Ohyama T. Detection system of Cryptosporidium parvum oocysts by brackish water benthic shellfish (Corbicula japonica) as a biological indicator in river water. Arch Environ Contam Toxicol 2006; 51: 559-66. doi:10.1007/s00244-0050174-9.

36.- Dedonno A, Liaci D, Bagordo F, Lugoli F, Gabutti G. Mytilus galloprovincialis as a bioindicator of microbiological pollution of coastal waters: a study conducted in the Salento Peninsula (Italy). J Coastal Res 2008; 24: 21621. doi:10.2112/05-0463.1.

37.- Reboredo Fernández A, Prado O, García T, Gómez Couso H, Ares E. Benthic macroinvertebrate communities as aquatic bioindicators of contamination by Giardia and Cryptosporidium. Parasitol Res 2014; 113: 1625-8. doi: 10.1007/s00436-014-3807-y.

38.- Vásquez Silva G, Castro Mejías G, González Mora I, Pérez Rodríguez R, Castro Barreara T. Bioindicadores como herramientas para determinar la calidad del agua. ContactoS 2006; 60: 41-8. https://agua.org.mx/wp-content/ uploads/2017/11/Bioindicadores-comoherramientas-para-determinar-la-calidad-delagua.pdf. 